\title{
IUE and ISO observations of the bipolar proto-planetary nebula Hen 401 (IRAS 10178-5958)^
}

\author{
M. Parthasarathy ${ }^{1,2}$, P. García-Lario ${ }^{3}$, G. Gauba ${ }^{2}$, D. de Martino ${ }^{4}$, Y. Nakada ${ }^{5,6}$, T. Fujii ${ }^{5}$, \\ S. R. Pottasch ${ }^{7}$, and L. San Fernández de Córdoba ${ }^{8}$ \\ 1 National Astronomical Observatory, 2-21-1 Osawa, Mitaka, Tokyo 181-8588, Japan \\ 2 Indian Institute of Astrophysics, Bangalore-560034, India \\ 3 ISO Data Centre, Astrophysics Division, Space Science Department of ESA, VILSPA, 28080 Madrid, Spain \\ 4 Osservatorio Astronomico di Capodimonte, Via Moiariello 16, 80131 Naples, Italy \\ 5 Institute of Astronomy, School of Science, University of Tokyo, 2-21-1 Osawa, Mitaka, Tokyo 181-8588, Japan \\ ${ }^{6}$ Kiso Observatory, School of Science, University of Tokyo, Mitake, Kiso, Nagano 397-0101, Japan \\ 7 Kapteyn Astronomical Institute, Postbus 800, 9700 AV Groningen, The Netherlands \\ 8 Laboratorio de Astrofísica Espacial y Física Fundamental, INTA, Apartado de Correos 50727, 28080 Madrid, \\ Spain
}

Received 6 April 2001 / Accepted 29 June 2001

\begin{abstract}
We present ultraviolet (IUE) and infrared (ISO) observations of the bipolar proto-planetary nebula Hen 401 which, combined with previously available optical and near infrared data, are used to reconstruct the overall spectral energy distribution from $1150 \AA$ to $100 \mu \mathrm{m}$. The ISO spectrum is dominated by strong PAH emission superimposed on a very cold continuum which is interpreted as thermal emission originating in the C-rich cool dust $(\sim 106 \mathrm{~K})$ present in the circumstellar envelope, the remnant of the previous AGB phase. In addition, a second, hotter component detected in the near infrared is attributed to thermal emission from hot dust $(\sim 640 \mathrm{~K})$, suggesting that mass loss and dust grain formation is still on-going during the current post-AGB phase. The ultraviolet (IUE) spectrum shows a stellar continuum in the wavelength interval $2400 \AA$ to $3200 \AA$ which corresponds to a moderately reddened B8-type central star. Unexpectedly, the UV flux in the wavelength interval $1150 \AA$ to $1900 \AA$ is very weak or absent with no evidence of a hotter binary companion which could explain the detection of the nebular emission lines observed in the available ground-based optical spectra of Hen 401. HST WFPC2 high resolution images also show no indication of a hot companion to the B8-type central star observed both in the optical and in the UV. The evolutionary implications of a possible single nature for the central star of Hen 401 are discussed.
\end{abstract}

Key words. planetary nebulae: individual: Hen 401 - ultraviolet: stars - stars: evolution - stars: emission-line stars: AGB and post-AGB

\section{Introduction}

Hen 401 is an early-type emission-line star (Henize 1976; Sanduleak \& Stephenson 1973) first identified as a post-asymptotic giant branch (post-AGB) star or a proto-planetary nebula (PPN) by Parthasarathy \& Pottasch (1989) on the basis of its far-infrared (IRAS)

Send offprint requests to: M. Parthasarathy,

e-mail: partha@iiap.ernet.in

* Based on observations obtained with the International Ultraviolet Explorer, retrieved from the INES Archive at VILSPA, Madrid, Spain, and observations made with ISO, an ESA project with instruments funded by ESA Member States (especially the PI countries: France, Germany, The Netherlands and the UK) with the participation of ISAS and NASA. colours. Parthasarathy \& Pottasch (1989) found the ratio of far infrared luminosity to visual luminosity to be very high and on the basis of this ratio suggested that the central B star may be obscured by a dusty disk or a flattened torus.

Hen 401 is a bipolar reflection nebula similar in morphology and spectrum to M 1-92. Recently Sahai et al. (1999) obtained high-resolution images of Hen 401 with the Wide Field Planetary Camera 2 (WFPC2) on board the Hubble Space Telescope (HST) detecting a highly collimated bipolar outflow. Two very long $\left(14.5^{\prime \prime}\right)$, cylindrical-shaped bipolar lobes are seen in reflected light, each with a length/width ratio of about 7 , probably the largest aspect ratio seen in a PPN so far. In the HST WFPC2 images the central star is resolved for the first 
time from the surrounding nebulosity. The HST images also revealed the presence of an equatorial torus and a bipolar skirtlike structure (see Fig. 1 in Sahai et al. 1999).

More than twenty years ago, Allen (1978) took the first optical spectrum of Hen 401 suggesting a Be spectral type for the central star. More recently, García-Lario et al. (1999; GL99 hereafter) obtained additional optical and near infrared spectra of Hen 401, confirming the detection of strong Balmer lines in emission followed in strength by [O I] emission and numerous Fe II and [Fe II] emission lines. Faint He I nebular emission lines were also detected in the spectrum, indicative of a hot central star, together with nebular emission lines due to [O II] (7319, $7330 \AA),[\mathrm{S} \mathrm{II}](4068,4076 \AA)$ and [N II] $(6584,6548$ and $5755 \AA$ ). The Ca II H and $\mathrm{K}$ lines, Ca II IR triplet lines and $[\mathrm{Ca} \mathrm{II}]$ lines, as well as some [Fe III] lines were also seen in emission. GL99 found a strong molecular emission line of $\mathrm{H}_{2}$ at $2.122 \mu \mathrm{m}$ which might have originated in the fluorescence-excited inner region of the circumstellar disk or by shocked-excited gas in the lobes.

Hen 401 was not detected in the $\mathrm{OH}$ maser emission line at $1612 \mathrm{MHz}$ by Silva et al. (1993). Instead, CO emission at millimeter wavelengths was detected by Loup et al. (1990) and Bujarrabal \& Bachiller (1991), suggesting a C-rich composition for the material ejected during the previous AGB phase. The expansion velocity of the molecular envelope derived from the $\mathrm{CO}$ emission lines was found to be $16 \mathrm{~km} \mathrm{~s}^{-1}$ (Loup et al. 1990). The CO emission is rather weak, which may be due to photodissociation by the UV radiation from the central star.

In Sect. 2 of this paper we present new observations of Hen 401 carried out in the UV (IUE) and in the infrared (ISO), which combined with previously available optical and near infrared data, are used to reconstruct the overall spectral energy distribution of Hen 401 from $1150 \AA$ to $100 \mu \mathrm{m}$. The main observational properties found are analysed in Sect. 3. Finally, in Sect. 4 we discuss the results obtained in the context of post-AGB stellar evolution as well as the nature of its central star.

\section{Observations}

\subsection{IUE data}

IUE low resolution ultraviolet spectra (1150 А to $3200 \AA$ ) of Hen 401 were obtained with the SWP and LWP cameras on board the IUE satellite using the large entrance aperture $\left(10^{\prime \prime} \times 23^{\prime \prime}\right)$. All spectra have been retrieved from the INES (IUE Newly Extracted Spectra) Archive, which contains spectra processed with the very recently improved IUE data extraction procedure (Rodríguez-Pascual et al. 1999, and references therein). Line-by-line images have been inspected for spurious features and identified. The $\log$ of the IUE observations is given in Table 1. The UV spectra of Hen 401 acquired in 1988 and in 1994 from $1150 \AA$ to $3200 \AA$ calibrated in absolute flux units are shown in Fig. 1.
Table 1. Log of the IUE observations.

\begin{tabular}{cccc}
\hline Camera & Image & Observation Date & Exposure Time(min) \\
\hline SWP & 33602 & 22 May 1988 & 110 \\
LWP & 13296 & 22 May 1988 & 50 \\
SWP & 50649 & 29 April 1994 & 180 \\
LWP & 28009 & 29 April 1994 & 60 \\
\hline
\end{tabular}

\subsection{ISO data}

A full range $2.4-45 \mu \mathrm{m}$ spectrum of Hen 401 was obtained on 1996 February 9 (TDT 08400730) using the SWS spectrograph (de Graauw et al. 1996) on board ISO (Kessler et al. 1996) as part of the guaranteed time proposal RWATERS.PAGB on spectroscopy of transition objects between AGB and PNe. The spectrum was taken in the standard Astronomical Observing Template (AOT) SWS01 mode with speed 1, which provides a spectral resolving power ranging from 400 to 600 .

On the same date another spectrum (TDT 08400731) was obtained this time with ISOPHOT-S, the spectrophotometer on board ISO (Lemke et al. 1996) covering the spectral range from 2.5 to $5 \mu \mathrm{m}$ (SS grating) and from 6 to $12 \mu \mathrm{m}$ (SL grating) with a spectral resolution of $0.0445 \mu \mathrm{m}$ and $0.0949 \mu \mathrm{m}$ respectively. The observations were made using the standard AOT PHT40 in staring mode under the same proposal time.

The SWS data, originally standard pipe-line processed with OLP version 8.4, were reprocessed using the latest version of the Observer's SWS Interactive Analysis (OSIA) software package available to date (version 1.0). All detector signals were inspected for spurious features (small jumps and glitches) which were removed. A flux depression originally observed in band $3 \mathrm{E}$ of the SWS spectrum was identified as an artifact produced by an incorrect dark current subtraction and, thus, the emission of this band was manually shifted to match the flux level of the adjacent bands $3 \mathrm{D}$ and 4 .

The PHT-S spectrum, also originally standard processed with OLP version 8.4 was further inspected for residual glitches not detected by the pipeline and corrected for transients using the newly available dynamic calibration method as it was implemented in the PHT Interactive Analysis (PIA) software package version 8.1. This method predicts the flux density of a target by direct comparison of the transient curve of the target itself with those of a set of standard stars (Laureijs et al. 2000), providing in most cases a final accuracy of $5-10 \%$.

\section{Analysis}

\subsection{UV continuum}

The UV continuum in the wavelength interval $1150 \AA$ to $1900 \AA$ is very weak or absent. However, in the LWP spectra a rising stellar continuum from $2400 \AA$ to $3200 \AA$ is clearly present (see Fig. 1). The Mg II $2800 \AA(\lambda \lambda 2795$, $2803 \AA$ ) line is found in emission and appears to show 


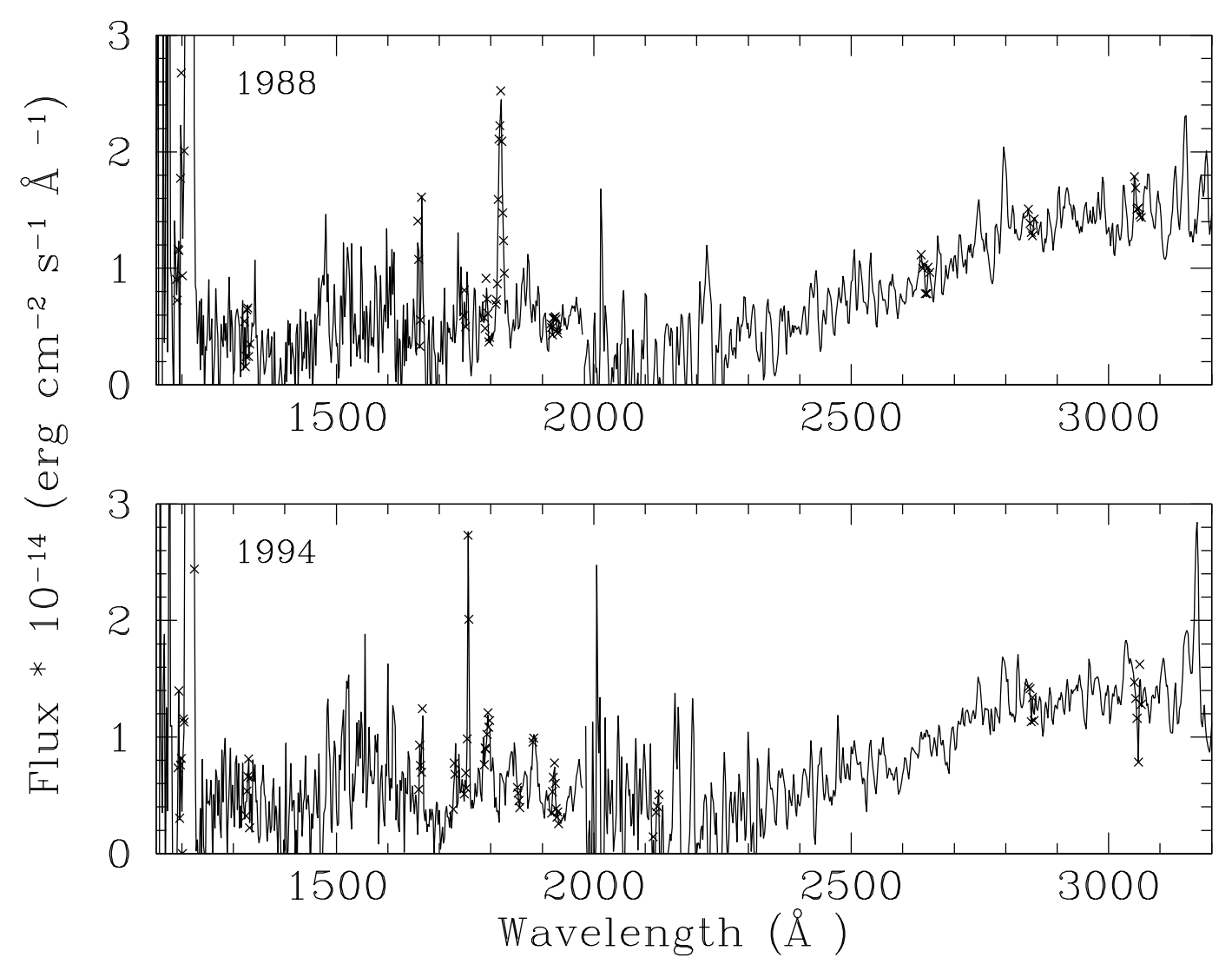

Fig. 1. SWP and LWP IUE spectra of Hen 401 in 1988 (top) and 1994 (bottom). Reseaux and spurious features identified in the line-by-line spectra are marked with crosses.

a decrease in strength. The Mg II $2800 \AA$ emission line fluxes in the 1988 and 1994 spectra are found to be $1 \times 10^{-12} \mathrm{ergs} \mathrm{s}^{-1} \mathrm{~cm}^{-2}$ and $7.7 \times 10^{-13} \mathrm{ergs} \mathrm{s}^{-1} \mathrm{~cm}^{-2}$ respectively. Within the uncertainties, there appear to be no other significant changes in the UV continuum between the 1988 and 1994 spectra.

The average UV continuum detected in the LWP spectra of Hen 401 was compared with the IUE spectra of a database of standard stars with different spectral types (Heck et al. 1984). After dereddening the IUE spectrum of Hen 401 using a value of $E(B-V)=0.43$ (see Sect. 3.2), we find that the LWP spectrum of Hen 401 closely resembles that of a B8 or an A0 star (Fig. 2).

The comparison made is illustrated in Fig. 2, which shows the average dereddened LWP spectrum of Hen 401 together with the dereddened spectra of HD 103287, a standard A0V star (upper panel) and of HD 222173, a standard B8V star (lower panel). Hence, we conclude that the central star of Hen 401 is more likely a late B or an early A star with no sign of a hotter binary companion in the UV.

\subsection{Reddening}

\subsection{1. $E(B-V)$ from $2200 \AA$ feature}

From an analysis of the $2200 \AA$ feature in the IUE spectra of Hen 401 we derive $E(B-V)=0.43 \pm 0.02$. This was

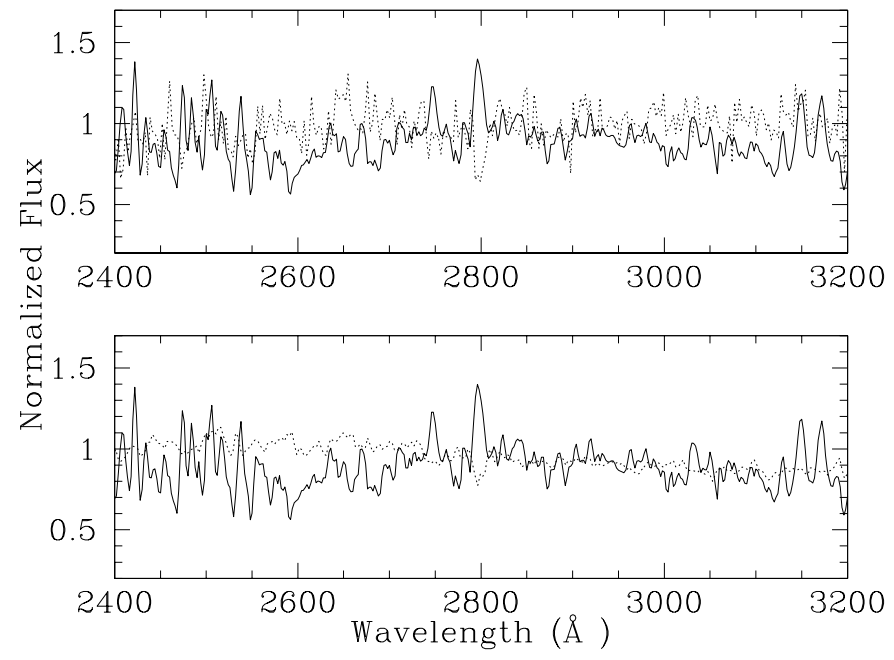

Fig. 2. The average LWP spectrum (solid line) of Hen 401 dereddened with $E(B-V)=0.43$ compared with that of HD 103287 (A0V) (upper panel) and HD 222173 (B8V) (lower panel).

made by fitting the $2200 \AA$ bump both using a rebinned $2 \AA$ spectrum and a $20 \AA$ spectrum with a reddened power law. This was done in order to get the the extinction without influences of spectral types. A similar extinction can be derived by making trials of dereddening with different 
values of $E(B-V)$. This way we confirm a value for the extinction of $E(B-V)=0.43$.

The study of circumstellar extinction around dusty carbon-rich and oxygen-rich objects is certainly very important. Muci et al. (1994) find that the extinction produced by the circumstellar dust is rather different. The circumstellar extinction toward carbon rich sources exhibits a broad absorption feature at wavelengths spreading from 2300-2500 ^ whereas the interstellar extinction feature at $2175 \AA$ seems to be unaffected (Muci et al. 1994). The carbon rich planetary nebula Abell 30, the carbon rich post-AGB star HD 213985 and the peculiar carbon rich object V348 Sgr show circumstellar extinction feature in the UV around $2500 \AA$ (see Greenstein 1981; Buss et al. 1989; Drilling \& Schönberner 1989 respectively). However, some of the carbon rich dusty post-AGB stars eg. HR 4049 (late B type star) and Red Rectangle (HD 44179) (late B type star) do not show the circumstellar extinction feature in the UV around $2500 \AA$ (Muci et al. 1994). Hen 401 also does not show the UV circumstellar extinction feature (2300-2500 ̊). Muci et al. (1994) find that the differences in the strength of $2300-2500 \AA$ feature in some of these stars could be attributed to the hydrogen content of the amorphous carbon grains responsible for the ultraviolet absorption.

The carbon rich post-AGB stars HR 4049 and the central star of Red Rectangle (HD 44179) have large amount of circumstellar dust and gas. However, the $2200 \AA$ feature in the UV spectra of these stars seems to be unaffected by the circumstellar reddening and the $E(B-V)$ estimated from this feature in the spectra of these stars seems to be mostly due to interstellar reddening (Buss et al. 1989; Sitko et al. 1981). From the ISO spectra of Hen 401 we find it to be a carbon rich post-AGB object and it appears to be similar to the Red Rectangle. The $E(B-V)$ estimated by us from the analysis of $2200 \AA$ feature in the spectra of Hen 401 is due to interstellar reddening.

The $2200 \AA$ absorption feature in the UV spectra of well known post-AGB stars HD 161796, HD 187885 (Parthasarathy et al. 1988) LS II $+34^{\circ} 26$ and LS IV $-12^{\circ} 111$ (Parthasarathy 1993; García-Lario et al. 1997a) and young PNe like SwSt1 (de Freitas Pacheco \& Veliz 1987) seems to be not affected by the circumstellar reddening. All these objects have large amount of circumstellar dust and some of them are carbon rich and some of them are oxygen rich. The $2200 \AA$ feature is not affected maybe because of the presence of large grains which produce neutral extinction or the circumstellar reddening law may be different from that of the interstellar reddening law.

\subsection{2. $E(B-V)$ from the Balmer emission lines}

In contrast, GL99 derived a much larger $E(B-V)=$ $1.3 \pm 0.4$ from the analysis of the Balmer decrement observed in the optical spectrum of Hen 401. We believe this value is probably an overestimation of the actual extinction if the strength of the Balmer lines is affected by collisional excitation, something which looks reasonable, given that many of the emission lines detected in Hen 401 are known to form only in very high density environments. Moreover, the Balmer lines in Hen 401 are suspected to show strong P-Cygni profiles which may also alter the observed Balmer line ratios. P-Cygni profiles are usually observed in objects showing strong bipolar outflows like Hen 401. Unfortunately, there is no high resolution spectrum yet available of the central star of Hen 401, but the possible presence of a strong P-Cygni profile affecting the measured strength of the Balmer lines is supported by the evident asymmetry observed in the profile of $\mathrm{H} \alpha$ in the low resolution optical spectrum shown in Fig. 2 of GL99.

\subsection{3. $E(B-V)$ from photometry}

An independent estimate of the extinction towards Hen 401 can be derived from the BVRI photometry obtained by Parthasarathy \& Ram Sagar (2001, in preparation) from broad band ground-based CCD images. The $B$ and $V$ magnitudes found are: $m_{B}=13.62, m_{V}=12.89$ with an estimated error of 0.03 in both filters. The observed $B-V=0.73$ is clearly inconsistent with the large $E(B-V)$ value derived from the Balmer decrement. Since the intrinsic $B-V$ colour is -0.11 for a B8 star and -0.02 for an $\mathrm{A} 0$ star we obtain a lower value of $E(B-V)=0.84$ or 0.75 , depending on the assumed spectral type. The $B$ and $V$ magnitudes derived from CCD images may be affected by the presence of emission lines in the optical spectrum of Hen 401, but the effect is expected to be very small according to the relative contribution of continuum and emission lines observed in the spectrum of Hen 401 in the wavelength range covered by these two filters.

In order to confirm that the presence of emission lines is not affecting our estimation of the extinction, an additional independent check was made considering this time only the continuum emission observed in the optical low resolution spectrum of Hen 401. By comparing this spectral continuum with that of standard stars of different spectral types using the library of stellar spectra compiled by Silva \& Cornell (1992) we again found it to be consistent with that of a B8-type star affected by an extinction of only $E(B-V)=0.85 \pm 0.10$ or an A0V star with $E(B-V)=0.75 \pm 0.10$, in very good agreement with the results obtained from the optical photometry.

The above results lead us to the conclusion that the extinction towards Hen 401 in the optical is close to $E(B-V)=0.85$ (assuming the B8 spectral type). It is significantly larger than $E(B-V)_{\text {interstellar }}=0.43$ derived from the $2200 \AA$ feature. The difference in the $E(B-V)$ values estimated from photometry and from $2200 \AA$ feature indicates that the circumstellar reddening $E(B-V)_{\text {circumstellar }}=0.42$.

Assuming that the extinction derived from the analysis of the IUE spectra is mostly interstellar in origin can be used to estimate the distance at which Hen 401 is located. A value of $E(B-V)=0.43$ for the interstellar 
extinction implies an interstellar contribution to the total visual extinction of 1.33 magnitudes. Hen 401 has a galactic longitude of 285.12 degrees and a latitude of -2.72 . Neckel \& Klare (1980) estimate a visual extinction of around 0.5 magnitudes per $\mathrm{kpc}$ at these galactic coordinates. Thus, we obtain an upper limit for the distance to Hen 401 of $2.66 \mathrm{kpc}$. Previous distance estimates were 3 or 4 kpc (Parthasarathy \& Pottasch 1989; Bujarrabal \& Bachiller 1991). In view of the uncertainities and complex nature of reddening and the central star of Hen 401 the above distance estimate should be used with caution.

\subsection{The ISO spectrum}

Figure 3 shows the ISO SWS spectrum (left) and the ISO PHT-S spectrum (right) of Hen 401. A first inspection reveals the presence of strong $\mathrm{PAH}$ features at 3.3, 6.2, 7.7, 8.6 and $11.3 \mu \mathrm{m}$, which confirms the C-rich nature of the circumstellar envelope.

The relative intensities of the detected $\mathrm{PAH}$ features are similar to those observed in other well-known C-rich PPNe like HR 4049 or IRAS 21282+5050 (Beintema et al. 1996). However, the 6.2, 7.7 and $8.6 \mu \mathrm{m}$ bands are slightly stronger than usual compared to the fainter $11.3 \mu \mathrm{m}$ band, suggesting that the emission is coming mostly from ionized PAHs (Pauzat et al. 1997) in Hen 401. This is also supported by the weakness of the $3.3 \mu \mathrm{m}$ band.

On the other hand, the non-detection of other $\mathrm{PAH}$ features sometimes observed longwards of $11.3 \mu \mathrm{m}$ (usually with peaks at 12.6 and $13.4 \mu \mathrm{m}$ or as a broad plateau of emission extending from 11 to $15 \mu \mathrm{m}$ ) suggests a low hydrogenation degree, associated with a more evolved stage in the transition from the post-AGB towards the PN phase.

It has been suggested that small cationic PAHs are the main carriers of DIBs (Salama et al. 1996). The presence of DIBs of circumstellar origin in the optical spectrum of Hen 401 (GL99) in combination with the detection of strong PAH emission in the ISO spectrum supports this connection.

In contrast with what has been observed in other C-rich PPNe like BD +30 3639 (Waters et al. 1998) or the Red Rectangle (Waters et al. 1999), no signature of crystalline silicates at $30-40 \mu \mathrm{m}$ is found in Hen 401 . The detection of crystalline silicates - an O-rich feature - in association with C-rich PPNe is usually associated either to the presence of a long-lived circumstellar disk or as a consequence of a recent episode of enhanced mass loss which produces a sudden increase of the dust temperature followed by a slow cooling process which favours crystallization as well as the survival of O-rich material in a C-rich environment.

\subsection{Spectral energy distribution}

In Fig. 4 we have combined the IUE spectrum of Hen 401 and the recently obtained BVRI photometry
(Parthasarathy \& Ram Sagar 2001, in preparation) with the available ground-based $J H K L^{\prime} M$ near infrared photometry (García-Lario et al. 1997b), IRAS photometry at $12,25,60$ and $100 \mu \mathrm{m}$ and ISO spectroscopy in order to reconstruct the overall spectral energy distribution of Hen 401 from the UV to the far infrared covering the whole spectral range from $1150 \AA$ to $100 \mu \mathrm{m}$.

The overall spectral distribution of Hen 401 can be well reproduced by a very simple model composed of three different black-bodies emitting at 106, 640 and $8500 \mathrm{~K}$. The coolest component is interpreted as thermal emission from the dust present in the cool, detached circumstellar envelope of Hen 401, the remnant of the previous strong mass loss AGB phase. The warm component at $640 \mathrm{~K}$ probably originates in the inner regions of the dusty circumstellar disk very close to the central star where hot dust grains (and PAHs) must have recently formed as a result of the on-going post-AGB mass loss. Finally, the third, hot stellar component detected in the UV and in the optical spectra, fitted by the $8500 \mathrm{~K}$ black-body continuum, must be the result of the contribution from the B8-A0 type central star.

\subsubsection{Energy budget}

The flux distribution of Hen 401 (Fig. 4) shows large near and far infrared excess due to warm and cold dust. The flux radiated in the near IR (from $1 \mu$ to $10 \mu$ ) is found to be $2 \times 10^{-9} \mathrm{ergs} \mathrm{s}^{-1} \mathrm{~cm}^{-2}$ and the far IR flux (12 $\mu$ to $100 \mu$ ) is found to be $7.62 \times 10^{-9} \mathrm{ergs} \mathrm{s}^{-1} \mathrm{~cm}^{-2}$. Whereas the flux radiated from $1400 \AA$ to $9000 \AA$ is found to be only $2.7 \times 10^{-10} \mathrm{ergs} \mathrm{s}^{-1} \mathrm{~cm}^{-2}$ after correcting for interstellar reddening $(E(B-V)=0.43)$. The flux ratio (near $\mathrm{IR}+$ far-IR) to that of flux from $1400 \AA$ to $9000 \AA$ is found to be about 36 . It indicates that the central star is heavily obscured by the dust. The near IR flux due to the warm dust (640 K dust component, Fig. 4) is 7 times more than that of the flux radiated by the star from $1400 \AA$ to $9000 \AA$. The total far-IR flux indicates the presence of large amount of cool dust. To account for the large ratio of far-IR flux to optical flux, we need a very bright and hot source at the center.

\subsubsection{Location of the dust with respect to the nebulosity}

The mid-IR imaging of PPNe indicates that the midIR emission arises mostly from the inner regions where the dust is warm. The far-IR emission $(>10 \mu)$ comes from the outer regions as well as from the inner regions. The hot dust (640 K, Fig. 4) is close to the central star and in the inner regions and the cool dust (106 K, Fig. 4) maybe confined to the outer regions. The HST images show that most of the Balmer emission and permitted forbidden emission lines are originating from the central $3^{\prime \prime}$ region (Sahai 1999). The mid-IR imaging of AFGL 2688 reveals that the warm dust is distributed 

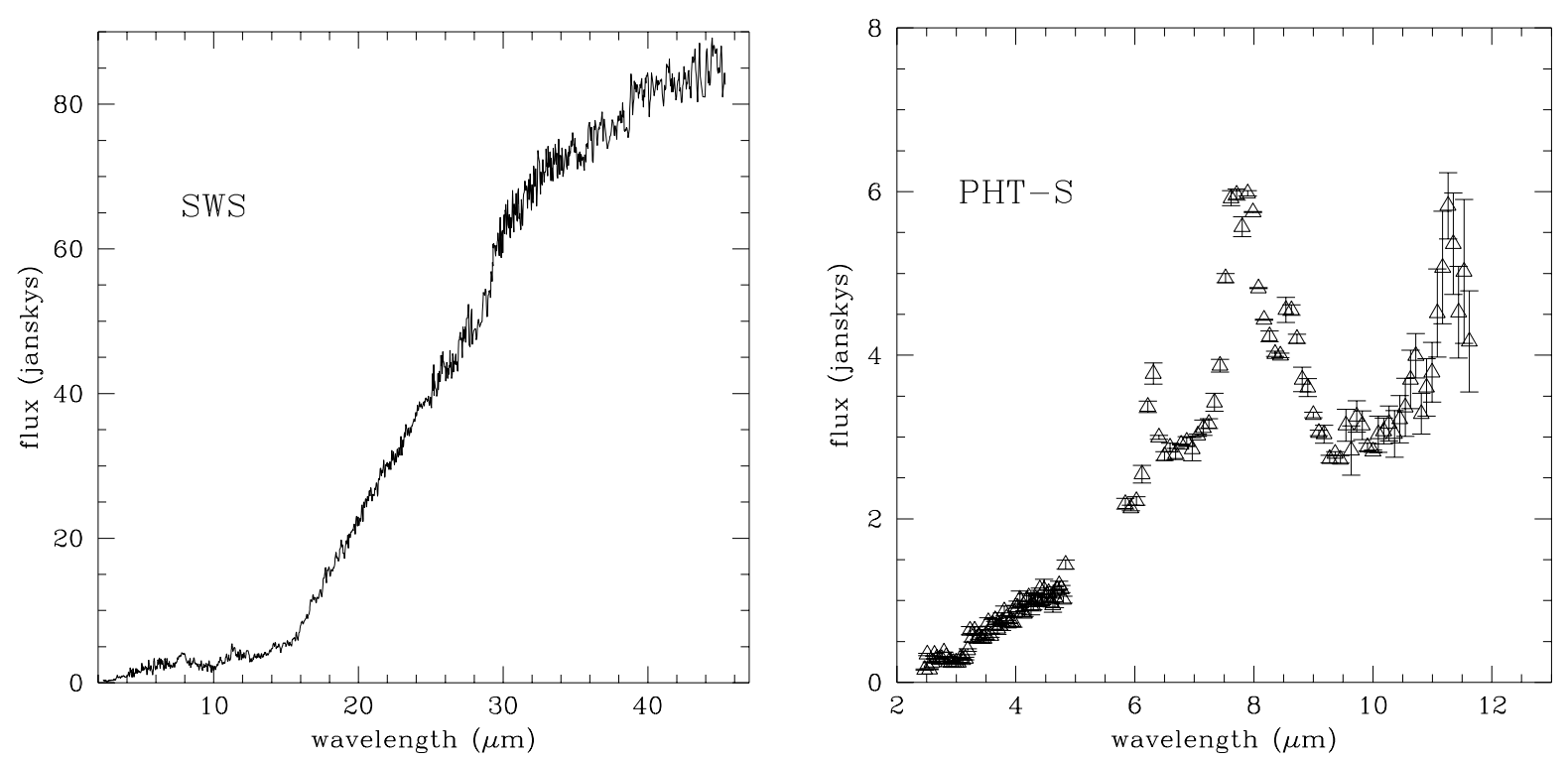

Fig. 3. ISO SWS (left) and PHT-S (right) spectrum of Hen 401.

predominantly along the polar lobes. Also weak mid-IR emission is found along the bipolar lobes (Morris \& Sahai 2000). In Hen 401 the bipolar lobes are limb brightened both in the visible and the near IR, indicating that the scattering optical depths are less than unity (from 0.6 to $2.2 \mu$ ). The emission produced in the bipolar lobes is indicative of shock heating. The molecular $\mathrm{H}_{2}(2.12 \mu)$ line emission is seen in the walls of the lobes but not from the skirt region. In contrast to the scattered light distribution which steadily falls with increasing distance from the central star, the $\mathrm{H}_{2}$ emission goes through a broad maximum (approximately $3^{\prime \prime}$ ) from the center. The imaging polarimetry of Hen 401 (Scarrott \& Scarrott 1995) shows a small elongated central nebula with a slight waist in the inner isophotes (about $2^{\prime \prime}$ north of the central brightness peak). This brightness structure, the circular polarisation pattern and the presence of a "polarisation disk" are all consistent with Hen 401 being a bipolar reflection nebula with a central illumination star which is heavily obscured from our direct view by a circumstellar disk. The HST images and the infrared data and the polarisation maps indiacate that the bulk amount of gas and dust is concentrated in the central region. Scarrott \& Scarrott (1995) also noted the presence of vestigial ansae on the major axis at $\pm 12^{\prime \prime}$ offset. The polarisation map of Hen 401 shows the presence of dust scattering all along the bipolar lobes with a total extent of more than $24^{\prime \prime}$. The polarisation map of Hen 401 shows similarities with that of Frosty Leo and Roberts 22 (Scarrott \& Scarrott 1995). Long slit optical spectroscopy and mid-IR high resolution imaging of Hen 401 may reveal more clearly the distribution of gas and dust.

\subsubsection{Effects of dust on the spectrum}

In order to further understand the effects of hot and cold dust on the optical and UV spectrum of Hen 401, we need to monitor it. Such a study has been carried out on some of the carbon rich post-AGB stars e.g. Red Rectangle (Waelkens et al. 1992; Sitko 1983; Sitko et al. 1981) and HR 4049 (Waelkens et al. 1991; Monier \& Parthasarathy 1999). High resolution spectroscopy of the late B central star of Hen 401 in the optical and ultraviolet may show depletion of refractory elements similar to that observed in the B9 central star of Red Rectangle and HR 4049 (Waelkens et al. 1992). The depletion of refractory elements and the normal abundance of volatile elements in these post-AGB stars is due to condensation of refractory elements into circumstellar dust grains. Long term monitoring of Hen 401 may show photometric variability in the ultraviolet and optical due to variable obscuration by circumstellar dust similar to that observed in the dusty post-AGB A type star HR 4049 (Waelkens et al. 1991; Monier \& Parthasarathy 1999).

\section{Discussion and conclusions}

The overall spectral energy distribution of Hen 401 confirms the classification of Hen 401 as a C-rich transition object in the PPN stage of evolution. It shows the characteristic double-peaked structure usually observed in other objects in the transition phase from the AGB to the PN phase (Parthasarathy \& Pottasch 1986; Kwok 1993).

ISO spectroscopy reveals a very cold continuum which is interpreted as being due to thermal emission from dust grains formed during the previous AGB phase. The low temperature of the dust in the shell indicates that the termination of the strong mass loss phase is not very recent. Such cold dust temperatures are only expected in well evolved PNe with detached dust shells.

In order to obtain information on the most recent postAGB mass-loss history one has to analyse the near infrared spectral range. Hot dust grains recently formed at temperatures close to the condensation limit $(\sim 1000 \mathrm{~K})$ 


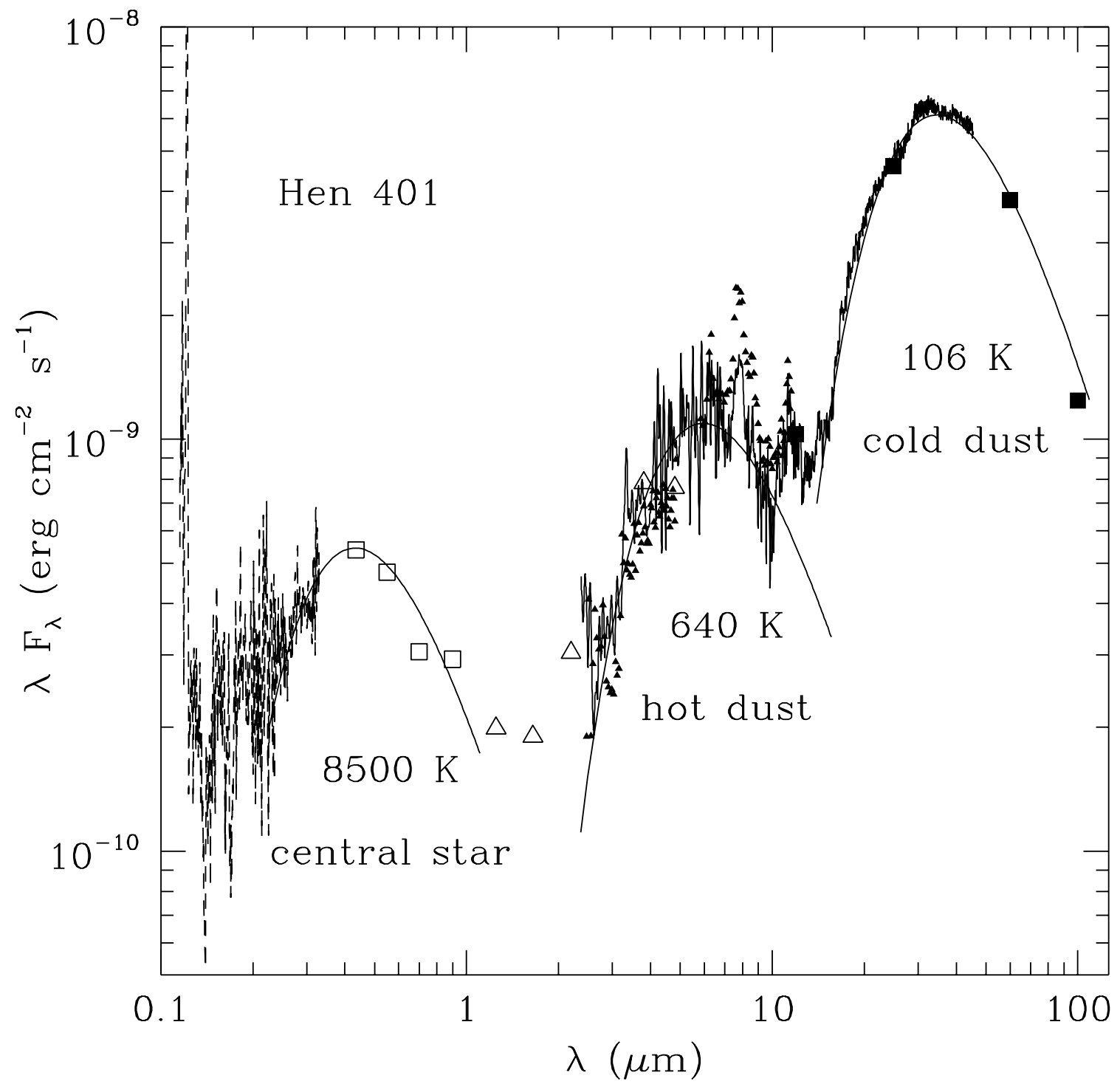

Fig. 4. The flux distribution of Hen 401 from the UV to the infrared (The data is corrected for interstellar reddening using $E(B-V)=0.43)$. ISO SWS (solid line), PHT-S (filled triangles) and IUE data (dashed line) are plotted together with groundbased optical $B V R I$ (open squares), near infrared $J H K L^{\prime} M$ (open triangles) and the IRAS photometry at $12,25,60$ and $100 \mu \mathrm{m}$ (filled squares).

are expected to emit thermally at these wavelengths. The near-mid infrared excess found in Hen 401 suggests that dust grain formation is still on-going in Hen 401 and that, although at a much lower rate than in the previous AGB phase, mass loss is still active during the current postAGB phase.

From a detailed analysis of the emission lines in the high resolution optical spectrum of Hen 401 we can infer the temperature of the central star. The presence of [N II], [S II] and He I emission lines and the absence of [O III] $5007 \AA$ suggests that the temperature of the post-AGB hot central star should be of the order of $22000 \mathrm{~K}$. This would imply an early-B spectral type for the central star of Hen 401, as already suggested by Henize (1976), Allen (1978) and Sanduleak \& Stephenson (1973). The same effective temperature is needed to explain the observed strength of the nebular Balmer emission lines. A similar result was obtained by Sahai et al. (1999) by comparing the HST images of Hen 401 in $\mathrm{H} \alpha$ and in the adjacent continuum.

However, the UV stellar continuum in the wavelength range $2400 \AA$ to $3200 \AA$ only indicates the presence of a late B- or early A-type star, not hot enough to explain the ionization observed in the optical or the detection of nebular emission lines. If we assume that this is also the star seen in the HST images (in the center of the lobes), the most natural explanation is that a hot post-AGB binary companion must exist, heavily obscured by a dusty disk or torus that seen almost edge-on in order to explain the non-detection of its strong UV flux with IUE.

One of the most important recent results is the recognition that fast $\left(>100 \mathrm{~km} \mathrm{~s}^{-1}\right)$ bipolar outflows can occur in proto-planetary nebulae. Objects like CRL2688, Red Rectangle and Hen 401 raise the question of how high 
velocity collimated flows and bipolar lobes can occur when the central star is cool (A or F spectral type; Frank 2000). The origin of the wind in these early stages and the mechanisms which produce collimation and bipolar lobes are critical questions. Interacting stellar winds scenarios generally invoke a dense wind or torus of gas near the central star in order to collimate the outflow that can account for the shapes of bipolar PNe. Simple interacting wind models are not able to explain the details seen in the high resolution HST images of bipolar PNe (Frank 2000). From theoretical models for shaping PNe (Frank 2000) it appears that hydrodynamic interacting stellar winds model may not be able to account bipolar PPN such as Hen 401. Magnetic fields may also play an important role in shaping up PPNe and PNe. It appears that much of the shaping and aspherical formation of PNe may occur during the proto-panetary nebula stage itself (Frank 2000; Frank 1999; Frank \& Mellema 1996).

The origins of bipolar structure in PNe are debated. In particular, whether the progenitor stars of bipolar nebulae most likely reside in binary systems or whether such nebulae can arise in the evolution of single stars. Morris (1981), Mastrodemos \& Morris (1998) suggest that the presence of a moderately close binary companion to a mass losing AGB star can account for almost all of the morphological characteristics of bipolar nebulae in PNe and PPNe. In the next few paragraphs we discuss binary system or single star as progenitor of Hen 401.

\subsection{Binary companion}

The detection of binary companions to the central stars of some other well-known bipolar PPNe like the Frosty Leo Nebula or the Red Rectangle (Roddier et al. 1995) has been used in the past to explain the development of bipolar structures around PPNe with disk or torus. In these binary systems the collimated bipolar outflows and jets are proposed to be the result of the direct or indirect interaction of the companion star.

The presence of a torus or a disk surrounding the central star can be inferred from the high resolution HST WFPC2 images of Hen 401 (Sahai et al. 1999). In addition, Scarrott \& Scarrott (1995) based on imaging polarimetry of Hen 401 found that the brightness structure, the circular polarization pattern and the presence of a polarization disk are all consistent with Hen 401 being a bipolar reflection nebula with a central illuminating star which is totally obscured from our direct view by a thick circumstellar disk. However, in the HST images we see a bright star which lies exactly midway between the nebular lobes in the centre of the bipolar structure which we identify with the B8 or A0 star detected in the optical spectrum and in the UV with IUE. If this star is the binary companion to a hot post-AGB central star which is so heavily obscured by the dusty disk that it is completely invisible in the HST images, it is difficult to explain how the star that we see in the optical is only affected by a modest circumstellar reddening. A possibility could be that this star is located just outside the line of sight of the dusty disk and hence it shows a low $E(B-V)$.

Discovering and studying binary central stars in $\mathrm{PNe}$ is very important to understand the common-envelope evolutionary phase, the origin of collimated bipolar outflows and to get distance estimates to PNe. Unfortunately, binary central stars are relatively rare in PNe and only a few of them are actually known to have resolved visual companions (Ciardullo et al. 1999). Bond (2000) lists $16 \mathrm{PNe}$ containing close binary nucleii.

\subsection{Single star scenario}

However, it is not always simple to determine the single or binary nature of central stars of PNe. Observationally, a number of them are known to be peculiar, as in the case of Hen 401, in that they have nebular emission lines in combination with the spectrum of a cool central star (Lutz 1977). Lutz proposed that at least some of them might represent single stars in a PPN stage of evolution. Spectral changes can be induced by sporadic post-AGB mass loss episodes which would favour the rapid increase of the stellar effective temperature (sometimes up to a level in which the nebular gas becomes ionized (e.g. Hen31357, SAO 85766) (Parthasarathy et al. 1993, 1995, 2000; Bobrowsky et al. 1998) followed by long periods (up to a few hundred years) of relative quiescence characterised by a progressive cooling of the central star. During the quiescence state an apparent discrepancy between the spectral type of the central star and the nebular conditions would be observable. Thus, we cannot completely rule out the possibility of the central star of Hen 401 being a single star until further observational confirmation is obtained.

The HST high resolution images (Sahai et al. 1999) reveal that the bulk of the emission, gas and dust and dense equatorial torus is within $2.8^{\prime \prime}$. Using the expansion velocity of $16 \mathrm{~km} \mathrm{~s}^{-1}$ derived from the millimetre $\mathrm{CO}$ observations (Loup et al. 1990) and adopting the angular diameter of the inner nebula and torus to be $2.8^{\prime \prime}$ (Sahai et al. 1999, GL99) we find the age of this structure to be 1107 years, if we assume the distance of $2.66 \mathrm{kpc}$ to be correct, which indicates that Hen 401 is a young bipolar PPN. Note that the long bipolar lobes with a total extent of $28^{\prime \prime}$ are expected to be the result of a very high velocity collimated post-AGB outflow (few hundred $\mathrm{km} \mathrm{s}^{-1}$ ) similar to that observed in some other bipolar PPNe and PNe such as M2-9, IRAS 17423-1755 (He 3-1475), KjPn 8 and $\mathrm{MyCn}$ 18. Assuming that the inner radius of the torus represents the end of AGB mass loss and that the high velocity collimated outflow began at the same time (using the $16 \mathrm{~km} \mathrm{~s}^{-1}$ expansion velocity derived from the $\mathrm{CO}$ emission) then the velocity of the bipolar lobes is of the order of $16 \times(28 / 2.8)^{\prime \prime}=160 \mathrm{~km} \mathrm{~s}^{-1}$. However, high resolution and long slit spectroscopy is required to determine the kinematical characteristics of the bipolar outflow and to compare with interacting stellar wind models. High resolution UV spectroscopy of the star seen at the center 
of the HST images may enable us to further understand the evolutionary status of Hen 401. Also mid-IR imaging may reveal the distribution and morphology of the dust.

Acknowledgements. We are thankful to the referee for helpful comments. We are thankful to Prof. Shuji Deguchi and Dr. R. Sahai for helpful comments and discussions. MP is very thankful to Prof. Shuji Deguchi, Prof. Norio Kaifu and Prof. Keiichi Kodaira for the kind encouragement, support and hospitality.

\section{References}

Allen, D. A. 1978, MNRAS, 184, 601

Beintema, D. A., van den Ancker, M. E., Molster, F. J., et al. 1996, A\&A, 315, L369

Bobrowsky, M., Sahu, K. C., Parthasarathy, P., \& GarcíaLario, P. 1998, Nature, 392, 469

Bond, H. E. 2000, in Asymmetrical Planetary Nebulae II: from Origins to Microstructures (ed. J. H. Kastner, N. Soker, \& S. Rappaport) ASP Conf. Ser., 199, in press

Bujarrabal, V., \& Bachiller, R. 1991, A\&A, 242, 247

Buss, R. H., Lamers, H. J. G. L. M., \& Snow, T. P. 1989, ApJ, 347,977

Ciardullo, R., Bond, H. E., Sipior, M. S., et al. 1999, AJ, 118, 488

de Freitas-Pacheco, J. A., \& Veliz, J. G. 1987, MNRAS, 227, 773

Drilling, J. S., \& Schönberner, D. 1989, ApJ, 343, 45

Frank, A., \& Mellama, G. 1996, AJ, 472, 684

Frank, A. 1999, New Astr. Rev., 43, 31

Frank, A. 2000, ASP Conf. Ser., 199, 225

García-Lario, P., Parthasarathy, M., de Martino, D., et al. 1997a, A\&A, 326, 1103

García-Lario, P., Manchado, A., Pych, W., \& Pottasch, S. R. 1997b, A\&AS, 126, 479

García-Lario, P., Riera, A., \& Manchado, A. 1999, ApJ, 526, 854

de Graauw, T., Haser, L., Beintema, D. A., et al. 1996, A\&A, 315, L49

Greenstein, J. L. 1981, ApJ, 245, 124

Heck, A., Egret, D., Jaschek, M., \& Jaschek, C. 1984, IUE LowDispersion Spectra Reference Atlas-Part I. Normal Stars (ESA SP-1052, ESTEC, Noordwijk, The Netherlands)

Henize, K. G. 1976, ApJS, 30, 491

Kwok, S. 1993, ARA\&A, 31, 63

Kessler, M. F., Steinz, J. A., Anderegg, M. E., et al. 1996, A\&A, 315, L27
Laureijs, R., Klaas, U., Richards, P. J., Schulz, B., \& Ábrahám, P. 2000, ISO Handbook 5: PHT - The Imaging PhotoPolarimeter SAI/99-069/Dc (version 1.1)

Lemke, D., Klaas, U., Abolins, J., et al. 1996, A\&A, 315, L64

Loup, C., Forveille, T., Nyman, L. A., \& Omont, A. 1990, A\&A, 227, L29

Lutz, J. H. 1977, A\&A, 60, 93

Mastrodemos, N., \& Morris, M. 1998, ApJ, 497, 303

Molster, F. J., van den Ancker, M. E., Tielens, A. G. G. M., et al. 1996, A\&A, 315, L373

Monier, R., \& Parthasarathy, M. 1999, A\&A, 341, 117

Morris, M. 1981, ApJ, 249, 572

Morris, M., \& Sahai, R. 2000, ASP Conf. Ser., 199, 143

Muci, A. M., Blanco, A., Fonti, S., \& Orofino, V. 1994, ApJ, 436,831

Neckel, T., \& Klare, G. 1980, A\&AS, 42, 251

Parthasarathy, M., \& Pottasch, S. R. 1986, A\&A, 154, L16

Parthasarathy, M., \& Pottasch, S. R. 1989, A\&A, 225, 521

Parthasarathy, M., Pottasch, S. R., \& Wamsteker, W. 1988, A\&A, 203, 117

Parthasarathy M. 1993, ApJ, 414L, 109

Parthasarathy, M., García-Lario, P., Pottasch, S. R., et al. 1993, A\&A, 267, L1

Parthasarathy, M., García-Lario, P., de Martino, D., et al. 1995, A\&A, 300, L25

Parthasarathy, M., García-Lario, P., Sivarani, T., Manchado, A., \& de Cordoba, S. F. 2000b, A\&A, 357, 241

Pauzat, F., Talbi, D., \& Ellinger, Y. 1997, A\&A, 319, 318

Roddier, F., Roddier, C., Graves, J. E., \& Northcott, M. J. 1995, ApJ, 433, 249

Rodríguez-Pascual, P. M., González-Riestra, R., Schartel, N., \& Wamsteker, W. 1999, A\&AS, 139, 183

Sahai, R., Bujarrabal, V., \& Zijlstra, A. 1999, ApJ, 518, L115

Salama, A., Bakes, E. L. O., Allamandola, L. J., \& Tielens, A. G. G. M. 1996, ApJ, 458, 621

Sanduleak, N., \& Stephenson, C. B. 1973, ApJ, 185, 899

Scarrott, S. M., \& Scarrott, R. M. 1995, MNRAS, 277, 277

Silva, A. M., Azcarate, I. N., Poppel, W. G. L., \& Likkel, L. 1993, A\&A, 275, 510

Silva, D. R., \& Cornell, M. E. 1992, ApJS, 81, 865

Sitko, M. L. 1983, ApJ, 265, 848

Sitko, M. L., Savage, B. D., \& Meade, M. R. 1981, ApJ, 246, 161

Waelkens, C., Lamers, H. J. G. L. M., Waters, L. B. F. M., et al. 1991, A\&A, 242, 433

Waelkens, C., Van Winckel, H., Trams, N. R., Waters, L. B. F. M. 1992, A\&A, 256, L15

Waters, L. B. F. M., Beintema, D. A., Zijlstra, A. A., et al. 1998, A\&A, 331, L61

Waters, L. B. F. M., Beintema, D. A., Cami, J., et al. 1999, in The Universe as seen by ISO, ed. P. Cox, \& M. Kessler, ESA SP-427, 219 